\title{
Supporting Information: \\ Evaporation of initially heated sessile droplets and the resultant dried colloidal deposits on substrates held at ambient temperature
}

\author{
Sanghamitro Chatterjee, Manish Kumar, Janani Srree Murallidharan and Rajneesh Bhardwaj* \\ Department of Mechanical Engineering, \\ Indian Institute of Technology Bombay, Mumbai, 400076 India \\ *Corresponding author (rajneesh.bhardwaj@iitb.ac.in, Phone: +91 222576 7534)
}

\section{Evaluation of average ring height and ring width from optical profilometer measurements}

The coffee-ring profile of the dried colloidal deposits was characterized by a 3D optical profilometer (Zeta 20, Zeta Instruments Inc., optical resolution $\sim 0.1 \mu \mathrm{m}$ ). The procedure for obtaining the average ring height and ring width, reported in figure 13 of the article is described below. The standard procedure for obtaining the average ring profile can be found elsewhere ${ }^{1,2}$, however, it is elaborated in this supporting information for the sake of completeness. Figure S1 (a) shows a representative microscopic view of a typical ring-like deposit obtained in the present study for a representative case of an initial droplet temperature of $25^{\circ} \mathrm{C}$ and suspended particle concentration of $0.1 \% \mathrm{v} / \mathrm{v}$. The ring profiles were measured for four different azimuthal locations (left, right, top, bottom) along a line around the ring as shown in figures 10-12 of the main article. The four azimuthal locations are shown by red boxes in figure S1 (a). The obtained profiles are plotted in figure S1 (b). An average ring profile was obtained from these profiles, which is shown by a thick black line in figure $S 1$ (b). The measured average ring profile is best fitted by a second-order polynomial curve using the least-squares fitting method. The average dimensions (average ring height and width) are obtained from the fitted curve, as shown in figure S2. 

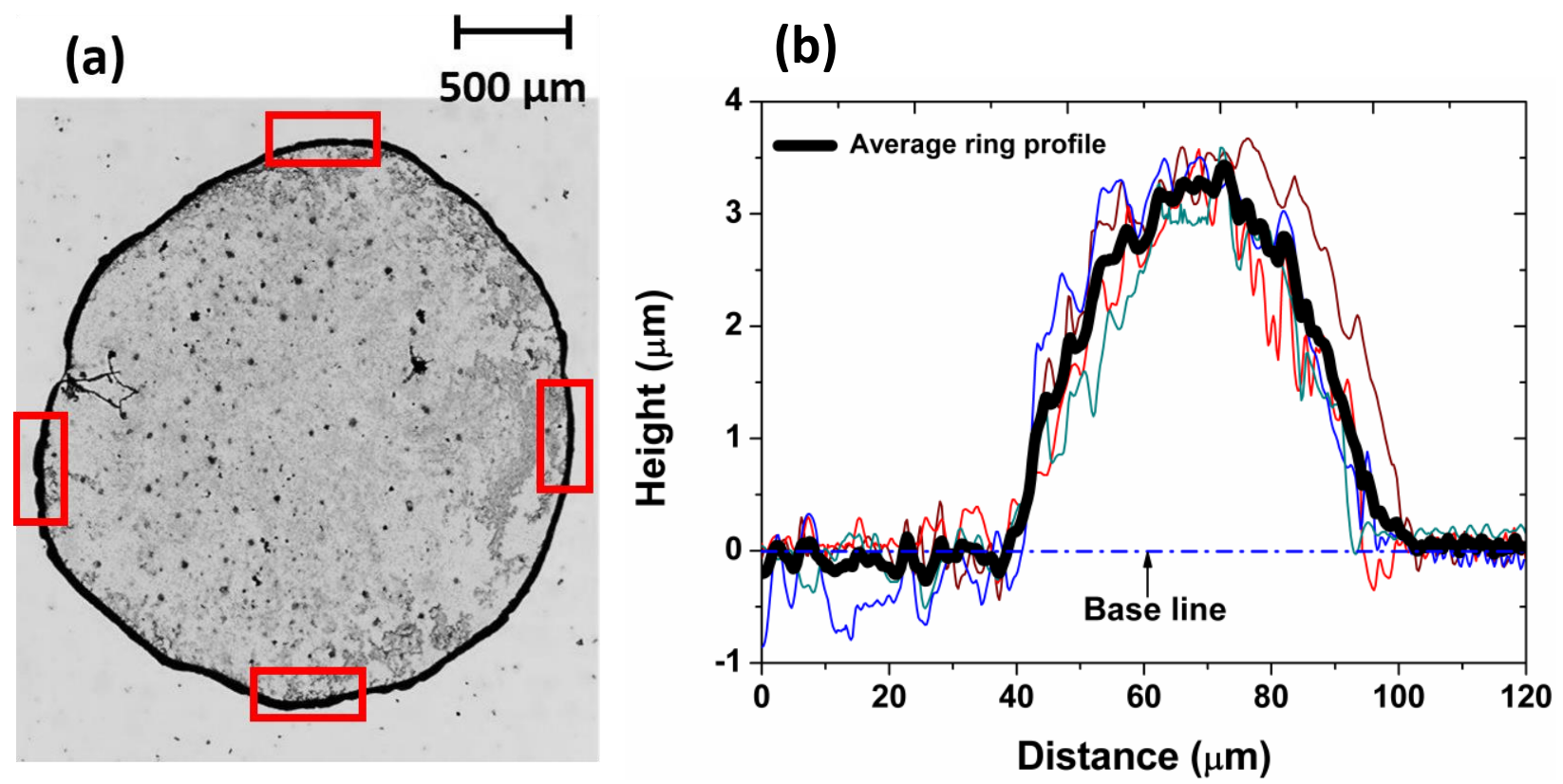

Figure S1. Method for obtaining average ring profile- (a) Microscopic view of a representative ring-like dried deposits after evaporation of a droplet having initial temperature equal to that of ambient and suspended particle concentration $0.1 \% \mathrm{v} / \mathrm{v}$. The four azimuthal locations at which the profiling is performed has been shown by red boxes. The profiling is performed along a line around the ring as shown in figures 10-12 of the main article. (b) Plots of ring profiles obtained at four different azimuthal locations shown in (a). An average of all these profiles is obtained which is shown by thick black curve in (b).

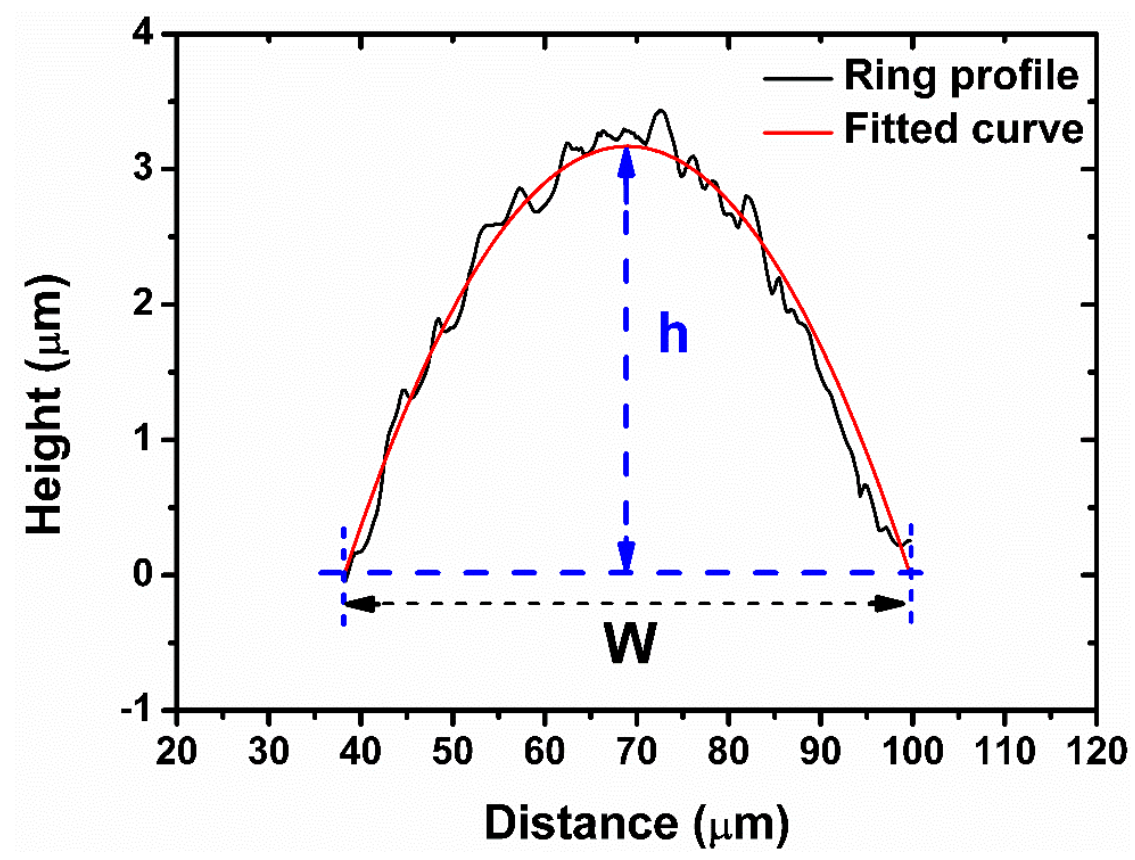

Figure S2. Polynomial fitting of average ring profile (cf. figure S1) for obtaining the average ring dimensions (height, $h$ and width $W$ ). 


\section{References}

1. Malla, L. K.; Bhardwaj, R.; Neild, A., Analysis of profile and morphology of colloidal deposits obtained from evaporating sessile droplets. Colloids and Surfaces A: Physicochemical and Engineering Aspects 2019, 567, 150-160.

2. Patil, N. D.; Bange, P. G.; Bhardwaj, R.; Sharma, A., Effects of Substrate Heating and Wettability on Evaporation Dynamics and Deposition Patterns for a Sessile Water Droplet Containing Colloidal Particles Langmuir 2016, 32, 11958-11972. 